with Papilocare $^{\circledR}$ (or extending it up to 12 -months if needed) in 3 out of 4 patients. These findings need to be confirmed upon study completion.

Disclosures Funding: Procare Health Disclosure: J.Cortés: Advisory/Consulting Role and Speaker at Procare Health. Y. Gaslain: CEO of Procare Health. All other authors have declared no conflicts of interest.

\section{EFFICACY OF A MULTI-INGREDIENT CORIOLUS VERSICOLOR-BASED VAGINAL GEL IN HIGH-RISK HPV+ PATIENTS: RESULTS OF DIFFERENT STUDIES}

${ }^{1}$ Yann Gaslain, ${ }^{2} J a v i e r$ Cortés, ${ }^{3}$ Damian Dexeus, ${ }^{4}$ Santiago Palacios, ${ }^{5}$ Clara Gajino, ${ }^{2}$ Giovanni Miniello, ${ }^{6}$ Margarita Riera, ${ }^{7}$ Elena Marin. ${ }^{1}$ Procare Health; ${ }^{2}$ Private Practice; ${ }^{3}$ Women's Health Institute; ${ }^{4}$ Instituto Palacios Salud Y Medicina de la Mujer; ${ }^{5}$ Hospital Materno Infantil Teresa Herrera; ${ }^{6}$ Institut Catala de la Salut; ${ }^{7}$ Hospital Alvaro Cunqueiro

\subsection{6/ijgc-2020-ESGO.2}

Introduction/Background To evaluate the consistency of the efficacy of a non-hormonal multi-ingredient Coriolus versicolor-based vaginal gel, Papilocare ${ }^{\circledR}$, on HPV clearance in patients infected by high-risk HPV (HR-HPV) in several studies.

Methodology Results at 6 months from independent observational non-comparative studies carried out in three different public centers and in a one private center were compared to results from both a randomized, open, parallel and controlled clinical trial comparing the Papilocare ${ }^{\circledR}$ vs wait and see approach (The Paloma RCT) and a observational, multicenter, prospective, one-cohort study (Papilobs real-life study). Two prospective (Vigo and Bari studies) and two retrospective studies (Coruña and Hospitalet studies) have been performed.

Vigo study: HPV clearance of 25 patients infected by HPV 16 and/or 18 was evaluated as a secondary endpoint.

Bari study: HPV clearance of 98 HR-HPV patients was evaluated as primary endpoint.

Coruña study: 57 medical records of patients with HRHPV were analyzed. HPV clearance was evaluated as primary endpoint.

Hospitalet study: Data of 91 HR-HPV patients were evaluated. Primary endpoint: composite efficacy variable (percentage of patients with normal cytology and/or HPV clearance).

Papilobs study: Interim data of $148 \mathrm{HR}-\mathrm{HPV}$ patients is presented. HR-HPV clearance was evaluated as secondary endpoint.

Paloma RCT: 66 HR-HPV patients were evaluated. Percentage of patients with HR-HPV clearance was assessed as a secondary endpoint.

Results After the 6-month treatment period, 48\% and 57\% of patients cleared HPV 16-18 and HR-HPV in Vigo and Bari studies, respectively. A reduction of $58 \%$ was observed in number of HR-HPV patients (Coruña) and $72.5 \%$ of patients negativized cytology and/or cleared HR-HPV (Hospitalet) $(p \leq 0.0001$ vs baseline for all results, Chi-square). In the Paloma RCT, HR-HPV clearance was observed in $63 \%$ of patients treated with Papilocare ${ }^{\circledR}$ vs $40 \%$ in the control group. Similar rate of 59\% HR-HPV clearance was observed in the interim analysis of the Papilobs study.

Conclusion Papilocare ${ }^{\circledR}$ has shown significant and consistent rates of HR-HPV clearance ranging from $50 \%$ to $70 \%$ in the 6 different studies. This high consistently rate of HR-
HPV clearance should be further confirmed in ongoing studies.

Disclosures Funding: Procare Health. Disclosures: J.Cortés, S. Palacios, D. Dexeus, L. Serrano: Advisory/Consulting Role and Speakers at Procare Health. Y.Gaslain: CEO of Procare Health. All other authors have declared no conflicts of interest.

\section{RATIONALE AND EVIDENCE FOR EMERGING ANTIBODY- DRUG CONJUGATES IN GYNECOLOGICAL CANCERS: EFFECT OF ONLINE EDUCATION ON CLINICIAN KNOWLEDGE}

${ }^{1}$ Geoff Fisher, ${ }^{1}$ Amy Furedy, ${ }^{1}$ Juliette Vandenbroucque, ${ }^{2}$ Nicole Concin. 'Medscape Global Education; ${ }^{2}$ Medical University of Innsbruck

10.1136/ijgc-2020-ESGO.22

Introduction/Background A number of targeted antibody drug conjugates (ADCs) are emerging with the potential to become important new treatment strategies for gynecological cancers, including recurrent/resistant ovarian and cervical cancers. This study determined whether online continuing medical education could improve the knowledge of oncologists and obstetricians/ gynaecologists (obs/gyns) on the rationale and evidence for emerging ADCs.

Methodology A 30-minute online video lecture was launched for physicians outside the USA in December 2019. Data was collected to March 2020. Educational effect assessed with repeated-pairs pre-/post-activity, where individual participants served as their own control. 3 multiple-choice, knowledge questions and 1 self-efficacy, 5-point Likert scale confidence question were analyzed. Chi-squared test assessed pre- to postactivity change $(5 \%$ significance level, $\mathrm{P}<.05)$. Magnitude of change in total number of correct responses overall, and for each question, were determined with Cramer's V $(<.06=$ Modest, $0.06-0.15=$ Noticeable, $.16-.26=$ Considerable, $>.26=$ Extensive).

Results 49 oncologists and 154 obs/gyns completed pre- and post-activity questions. A positive educational effect was observed for oncologists (considerable effect, $\mathrm{V}=.217$, $\mathrm{P}=.0002$ ) and obs/gyns (noticeable effect, $\mathrm{V}=.097, \mathrm{p}=.0028$ ) with average $\%$ of correct responses increasing 40 to $62 \%$ for oncologists and 34 to $43 \%$, for obs/gyns. Participants with $3 / 3$ answers correct increased from pre- to post-activity (6 to $35 \%$ for oncologists and 6 to 12\%, for obs/gyns). Improvements in $\%$ of correct responses post-activity were seen for all 3 knowledge-based questions on antigen targets, and key trial data for tisotumab vedotin and mirvetuximab vedotin $(88 \%$, $39 \%$, 45\% improvements for oncologists; 70\%, 15\%, 16\% improvements for obs/gyns). Confidence in knowledge of ADCs also improved post-activity with a total average confidence shift of $38 \%$ for oncologists and $32 \%$ for obs/gyn. $62 \%$ of oncologists' and $44 \%$ of obs/gyns' responses were reinforced or improved post-activity. 34\% of all participants stated they would modify treatment plans as a result of participation in the activity.

Conclusion This on-demand, online video lecture resulted in a positive education effect for both oncologists and obs/gyns. However, persistent knowledge gaps are evident, especially amongst obs/gyns, suggesting there is a need for additional education as data on ADCs continues to emerge. Online medical education is valuable in establishing improved knowledge 